\title{
TSR: A STORAGE RING FOR HIE-ISOLDE*
}

\author{
P.A. Butler, R.D. Page
}

Oliver Lodge Laboratory, University of Liverpool, Liverpool L69 7ZE, UK

K. Blaum, M. Grieser

Max-Planck-Institut für Kernphysik, 69117 Heidelberg, Germany

T. Davinson, P.J. Woods

School of Physics and Astronomy, University of Edinburgh

Edinburgh EH9 3JZ, UK

K. Flanagan, S.J. Freeman

School of Physics and Astronomy, University of Manchester

Manchester M13 9PL, UK

I.H. LAZARUS

S.T.F.C. Daresbury Laboratory, Warrington WA4 4AD, UK

$$
\text { Yu.A. Litvinov }
$$

GSI Helmholtzzentrum für Schwerionenforschung, 64291 Darmstadt, Germany

$$
\text { R. RAABE }
$$

KU Leuven, Instituut voor Kern- en Stralingsfysica, 3001 Leuven, Belgium

E. Siesling, F. Wenander

CERN, 1211 Geneva 23, Switzerland

(Received November 17, 2015)

It is planned to install the heavy-ion, low-energy ring TSR, currently at the Max-Planck-Institute for Nuclear Physics in Heidelberg, at the HIEISOLDE facility in CERN, Geneva. Such a facility will provide a capability for experiments with stored, cooled secondary beams that is rich and

* Presented at the XXXIV Mazurian Lakes Conference on Physics, Piaski, Poland, September 6-13, 2015. 
varied, spanning from studies of nuclear ground-state properties and reaction studies of astrophysical relevance, to investigations with highly-charged ions and pure isomeric beams. In addition to experiments performed using beams recirculating within the ring, the cooled beams can be extracted and exploited by external spectrometers for high-precision measurements. The capabilities of the ring facility as well as some physics cases will be presented, together with a brief report on the status of the project.

DOI:10.5506/APhysPolB.47.627

\section{Introduction}

In order to address many key questions in nuclear science, measurements of nuclear reactions and properties of unstable nuclei must be studied with a precision sensitive to their underlying quantum structure. Final states of interest often lie in regions of excitation energy with high level density and thus energy resolution is a critical issue, which is determined both by the beam characteristics and by the detector design and performance. Additionally, access to a wide range of radioactive beam species is critical for the scientific reach of the programme allowing the probing of key nuclei and regions of astrophysical importance, and where new shell structures and shape phenomena may be discovered. The ISOLDE facility at CERN, that produces a wide range of intense radioactive beams, is being upgraded (HIE-ISOLDE) to accelerate these beams to $5.5 \mathrm{MeV} A$ by 2016 and $10 \mathrm{MeV} A$ in the following two years. It is planned to install a heavy-ion storage ring (TSR) at ISOLDE. This unique facility will allow the vast range of isotopes to be injected into the ring at energies ideal for the science programme. Cooling within the storage ring will produce radioactive beams of extremely high quality with a factor of ten better transverse emittance and energy spread compared to the direct beam from HIE-ISOLDE. In addition, beams stored in the TSR can result in significant beam purification because of the low momentum spread $\left(1\right.$ in $\left.10^{5}\right)$ of the cooled beams, which can be harnessed to produce pure isomeric beams. A Technical Design Report on the technical aspects of the implementation of the TSR at CERN and the scientific opportunities has been published [1].

\section{Injection and measurement cycles}

The way to achieve an efficient transfer of the ion beam into the TSR at Heidelberg is by occupying the available phase space through a 'multi-turn' injection. This operation is fast $(\approx 30 \mu$ s using 25 turns at a beam energy of $10 \mathrm{MeV} A$ ), however, it has to be followed by a cooling time during which the beam is reduced in energy spread and size, from a diameter of a few centimeters to about $1 \mathrm{~mm}$. This is achieved with electron cooling. Cooling 
also reduces the transverse emittance and momentum spread of the recirculating ions by factors of 10 or more, see figure 50 in [1]. Cooling times may vary between about $0.2 \mathrm{~s}$ for $A=200$ with $A / q=4$ and about one second for $A=25$ with $A / q=2.5$. When cooling is complete, a measurement can take place in the TSR, until the following injection. Alternatively, several injection-cooling cycles can follow each other increasing the particle current, before performing the measurement. The range of $A / q$ and beam magnetic rigidity provided by HIE-ISOLDE is well-matched to the TSR (see figure 18 in [1]), although an upgrade to the EBIS charge breeder will be required to facilitate the injection of the heaviest ions. The injection of highly-stripped ions from an upgraded EBIS will also enhance the survival of the recirculating ions that would otherwise undergo charge exchange in the residual gas or gas-jet target. This will increase the storage time from $1 \mathrm{~s}$ to $10 \mathrm{~s}$ for the heavier $(Z>40)$ ions for a $\mathrm{H}_{2}$ target having a density of $3 \times 10^{13}$ atoms $/ \mathrm{cm}^{2}$.

\section{In-ring reaction measurements}

Lighter mass beams can be stacked and recirculated through an ultrathin, ultra-pure internal gas target. High luminosity can be achieved since the beam has multiple $(\sim 1 \mathrm{MHz})$ passes through the target. Light reaction products can be observed using an internal spectrometer in the ring, consisting of an Si array adapted for UHV conditions with recoil products detected in a downstream detector. The technical challenge for the in-ring spectrometer is to detect outgoing light particles from reactions, such as $(d, p)$ and $(p, d)$, for which the differential cross sections in the centre-of-mass frame peak at small scattering angles. In inverse kinematics, this corresponds to the detection of light particles with energies less than $\approx 10 \mathrm{MeV}$ at laboratory angles dependent on the reaction. For example, forward laboratory angles near $0^{\circ}$ correspond to the peak cross sections for $(p, d)$, but backward laboratory angles for $(d, p)$. In either case, the detector array will have to be mounted on actuators so that the detectors are withdrawn during the beam injection phase, when the beam diameter is large (a few $\mathrm{cm}$ in diameter) prior to cooling (see figure 1). The forward angles in the centre of mass are generally most important for studies of astrophysical resonances, since the critical states are produced in low orbital angular momentum transfer reactions (e.g. s- and p-wave transfers) which peak in cross section at forward angles. In stellar burning conditions, the most important states correspond to resonances with low centrifugal barriers and, therefore, high quantum tunnelling probabilities. In addition to the detectors positioned closest to the target region, particle detection at and around $0^{\circ}$ in the laboratory frame will be needed for other reactions of interest (e.g. proton-capture reactions) and for detection of the heavy projectile-like fragments at for- 

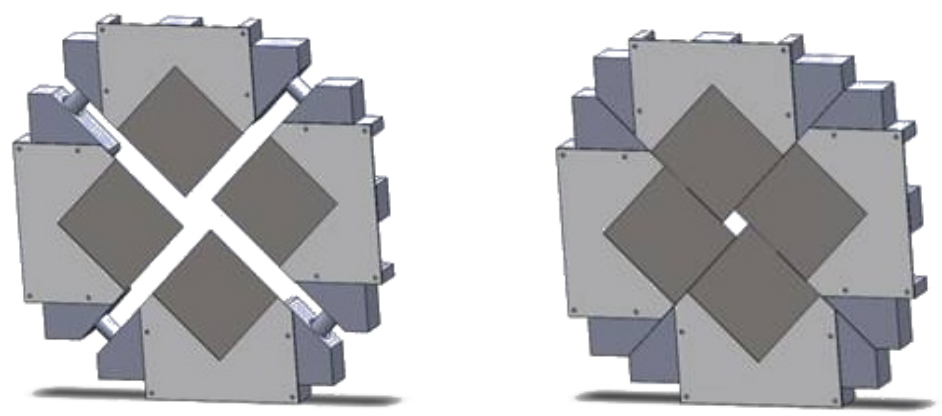

Fig. 1. Schematic layout of the in-ring Si detector array mounted on a 4-jaw actuator. The left figure shows the configuration when the beam is injected, the right figure when the beam is cooled.

ward angles. Compared to conventional approaches, in-ring experiments for lighter mass beams will benefit from increased luminosity from recirculation and improved resolution from a high-quality beam. In order not to degrade the potential resolution, particle detection should be performed with minimal intervening material, necessitating thin window-less gas-jet targets and thin detector dead layers. Excellent angular resolution is necessary, to minimise the effects of kinematic broadening, combined with a high intrinsic energy resolution in the detector to obtain the highest precision and sensitivity for the excitation-energy spectrum (corresponding to the ion energy in the centre-of-mass frame).

The provision of a detection system in the ring provides many opportunities for applications to nuclear astrophysics. For example, cataclysmic astronomical events, such as supernovae, neutron star mergers, and novae, are now generally understood to constitute the source of almost all the chemical elements we find on Earth and observe in our Galaxy. However, the specific stellar production site for a vast majority of elemental species is still largely undefined, due to uncertainties in the key underlying nuclear physics processes that drive explosive stellar scenarios. In particular, $(p, \gamma)$ and $(\alpha, \gamma)$ radiative capture reactions on unstable nuclei play a critical role, determining both the rate of energy release and path of nucleosynthesis in such events [2]. Unfortunately, nearly all of these key astrophysical reactions are too weak to be measured directly in the relevant low-energy Gamow burning regime and consequently, an indirect approach is required for progress to be made. Single-nucleon transfer reactions probe the basic single-particle structure of nuclei and may be used to populate excited states, corresponding to astrophysical resonances in $(p, \gamma)$ and $(\alpha, \gamma)$ reactions. In particular, these studies allow for the extraction of spin-parity assignments and spectroscopic factors for key resonant states. By obtaining a measure of such 
properties, it is possible to indirectly determine the stellar reaction rate (the rate is directly proportional to the strength of each resonance, while the strength depends on the spin-parity assignment and spectroscopic factor of the state). In the case of explosive astrophysical reactions, where radioactive nuclei are involved, transfer reactions must be performed in inverse kinematics. This traditionally generates a number of experimental challenges but, in fact, makes the use of the TSR uniquely well-suited. Specifically, a key experimental aspect is that astrophysical resonances often exist in regions of high level-density where excited states are present every $30-40 \mathrm{keV}$ and as such, ultra-high resolution spectroscopy is essential. In many instances, one is dealing with relatively light heavy-ion beams, which can easily be produced fully stripped, and which have lifetimes greater than 1 second, since, in general, the most important individual reactions involve radioactive isotopes close to, or adjacent to, the valley of $\beta$-stability. Therefore, it is possible to use such beams for reaction studies within the TSR. The use of high-quality, pure radioactive-ion beams from the TSR in combination with a newly designed, highly segmented silicon detection system will allow for ultra-high resolution to be achieved.

\section{External spectrometer}

Figure 2 shows the variation of the product of luminosity and solid angle as a function of mass for a typical internal spectrometer configuration. The luminosity will be determined by the storage time and the nuclear lifetime, whichever is the shorter. For masses $>100$, charge exchange effects



Fig. 2. Variation of the product of luminosity and solid angle with mass for the in-ring detector array. The luminosity is mostly determined by the storage time of the recirculating ion undergoing charge-exchange in the deuterium gas jet target, having a thickness of $10^{14}$ atoms $/ \mathrm{cm}^{2}$. This quantity is compared with that of the HELIOS spectrometer, using a target thickness of $60 \mu \mathrm{g} / \mathrm{cm}^{2}$. 
in the gas-jet target reduce the luminosity to the point where it is better to extract the beam into an external spectrometer. Here, the light-particle reaction products will be transported in helical orbits in a uniform magnetic field to a linear Si array positioned along the beam axis in a superconducting solenoid; this is the HELIOS concept $[3,4]$. The light-particle reaction products return to the beam-axis where they are measured in the positionsensitive Si detector array. The measured experimental parameters are the distance from the target at which the ion returns to the beam axis $(z)$, the energy of the ion in the lab frame $\left(E_{\text {lab }}\right)$ and the time of flight of the ion $\left(t_{\mathrm{cyc}}\right)$. The latter corresponds to the cyclotron period of the ion in the field. The distance and energies are obtained from the Si array. Forwardgoing heavy recoils are detected to provide a time reference to obtain $t_{\mathrm{cyc}}$, enabling the mass-to-charge ratio of the ion to be determined [3]. Measurements of outgoing ion energies in the laboratory must be transformed into the centre-of-mass frame to obtain the $Q$-value or excitation-energy spectrum. In a solenoid system, the energy in the centre-of-mass frame, and therefore reaction $Q$ value, for ions with a particular value of $z$ is a linear function of $E_{\text {lab. }}$. This compares to conventional systems, where detectors are placed at particular scattering angles and the $Q$ value is a complex nonlinear function of laboratory angle and energy. The planned spectrometer will allow measurements with $Q$-value resolutions down to $\approx 20 \mathrm{keV}$. The $\mathrm{Si}$ array will use double-sided silicon detectors, rather than resistive readout, to give $1 \mathrm{~mm}$ position resolution in the axial direction and $2 \mathrm{~mm}$ in the transverse direction. This allows the extrapolation of the particle trajectory to the beam axis itself. This is necessary because the Si array has a finite diameter, and the interception of the trajectory with the detector happens at a smaller distance from the target than the interception on the beam-axis. The contributions to the expected $Q$-value resolution using the extracted TSR beam are much smaller than that obtained using direct beams from HIE-ISOLDE. For a typical reaction such as $d\left({ }^{132} \mathrm{Sn}, p\right){ }^{133} \mathrm{Sn}$ at $10 \mathrm{MeV} A$ the $Q$-value resolution improves by a factor of two, approaching $20 \mathrm{keV}$.

As just one example, around $N=82$ neutron-adding reactions on stable targets have been used to investigate the trends in high- $j$ single particle strengths. The fragmentation of $\nu i_{13 / 2}$ and $\nu h_{9 / 2}$ states is quite high, due to extensive coupling with vibrational excitations of the core. Measured spectroscopic factors are essential to disentangle the trends in single-particle energies from this coupling as a function of proton number. The separation of the resulting centroid energies of these single-neutron states monotonically decreases with increasing proton number, a variation that appears to be consistent with the effects of the phenomenological tensor interaction between the neutrons in the $i_{13 / 2}$ and $h_{9 / 2}$ states and protons filling mainly the $g_{7 / 2}$ orbital $[5,6]$. Away from stability, at higher proton numbers, there is the expectation that as the proton Fermi surface moves from filling $\pi g_{7 / 2}$ 
to $\pi h_{11 / 2}$, the sense of the tensor interaction will reverse causing the difference in energy of the $\nu i_{13 / 2}$ and $\nu h_{9 / 2}$ neutron states to begin to increase. Indeed, the energies of the lowest $13 / 2^{+}$and $9 / 2^{-}$states do suggest such a turnaround, but there is no information on the spectroscopic factors away from stability. In fact, it is expected that the coupling to octupole vibrations will be highest at $Z=64$, exactly where the trend reverses, meaning that the energies of the lowest states are likely to be a very poor indicator of the single-particle energies.

In order to resolve these issues, measurements are needed of spectroscopic factors with beams such as ${ }^{146} \mathrm{Gd}$ and heavier $N=82$ species. Such studies would employ $(d, p)$ reactions, or for improved matching for high $\ell$ transfer, the use of $\left(\alpha,{ }^{3} \mathrm{He}\right)$ reactions would be an advantage. Similarly, the shell evolution can be mapped out around $Z=82$, using $\mathrm{Pb}$ beams, or $N=126$, using beams such as ${ }^{206} \mathrm{Hg}$ and heavier. Previous measurements have mostly used traditional spectrometers (such as Enge splitpole) to analyse the reaction products; more recent measurements have used HELIOS [6] with stable beams and $\mathrm{Si}-\mathrm{Ge}$ low-resolution arrays for radioactive ion beams [7]. For low-intensity radioactive beams the use of a spectrometer of high efficiency and good energy resolution such as the system described here is highly desirable.

Another example of the external spectrometer coupled to the TSR is the study of heavy pear-shaped nuclei. Future experiments at the HIE-ISOLDE facility will study ${ }^{222,228} \mathrm{Ra}$ and ${ }^{222-226} \mathrm{Rn}$, which should greatly enhance our knowledge of the systematic behaviour of E3 moments in this mass region [8] and help us to assess the likelihood of parity doubling in the odd- $A$ nuclei. There are also plans to measure directly the low-lying structure of odd-



Fig. 3. Expected spectrum of states in ${ }^{225} \mathrm{Ra}$ populated by the $\left(d, d^{\prime}\right)$ reaction at a bombarding energy of $10 \mathrm{MeV} A$. The relative intensity of the low-lying states, labelled by their spin, parity and $\ell$ transfer, is given. Superimposed on the spectrum is the expected energy resolution of the particle spectrometer. 
mass $\mathrm{Rn}$ isotopes, from in-beam conversion-electron and $\gamma$-ray measurements following Coulomb excitation or from the decay of the astatine parent. These measurements are vital in order to identify the best candidates for the radon EDM programme. More challenging, however, is the measurement of the inter-band E3 matrix elements between the low-lying states in odd-mass nuclei, required for both radon and radium EDM experiments. This will require advances in precision spectroscopy. An approach advocated here is to employ $\left(d, d^{\prime}\right)$ scattering (see, e.g. [9]) to populate low-lying states in odd- $A$ nuclei via single-step excitation. We have carried out simulations that indicate that $\sim 20 \mathrm{keV}$ energy resolution for the final states can be reached by using the HELIOS-type spectrometer, provided that the radioactive heavy beams are cooled in a storage ring such as the TSR. The expected spectrum of states in ${ }^{225} \mathrm{Ra}$ populated by such a reaction is shown in figure 3 .

\section{In-ring decay measurements and laser spectroscopy}

The potential application of the TSR to in-ring decay measurements is extensively documented in [1]. For measurements of $\beta$-decay to unbound states in exotic light nuclei, the emitted particles having small centre-ofmass energies can be easily detected in-flight in the ring. The emission cone will be small (a few degrees), but the laboratory energy will be of the order of $10 \mathrm{MeV}$. There are several examples where this technique could be applied, e.g. the study of $\beta$-delayed proton emission of the halo nucleus ${ }^{11} \mathrm{Be}$ [10].

A second application of in-ring decay [see [1] for more details] is the measurement of the lifetime of hydrogen-like ${ }^{7} \mathrm{Be}$. It is estimated that $20 \%$ of ${ }^{7} \mathrm{Be}$ in the centre of the Sun has a bound electron, and an accurate measurement of the lifetime of this state would allow, for example, for a deeper insight into the branchings within the proton-proton chain reaction in the Sun, knowing the overall ${ }^{7}$ Be neutrino flux.

The provision of a recirculating radioactive ion beam provides a third application: collinear laser spectroscopy. Light elements are difficult to study using traditional laser spectroscopy for a number of reasons [11]. They are produced in ISOL mainly as molecules unsuitable for studying hyperfine effects. The calibration of charge-radii measurements is difficult with the relatively few stable systems where radii are determined in other ways, and theoretical atomic models of multi-electron atoms are not sufficiently precise to provide that calibration. In some cases, the necessary wavelengths are not obtainable using current laser systems. The TSR presents an excellent opportunity to study difficult cases previously inaccessible at ISOL facilities and provides several advantages over existing laser spectroscopy techniques (see figure 4). Molecular ions are broken up as part of the HIE-ISOLDE accelerator system (in REX-EBIS) that can produce highly charged heliumlike ions. The latter are suitable for laser spectroscopy and can be calculated with high precision in atomic models for radii calibration. Acceleration 
allows some inaccessible transition energies to be Doppler shifted into the range of current laser technology, whereas recirculation in the TSR allows multiple laser excitation increasing the apparent luminosity for weak beams.

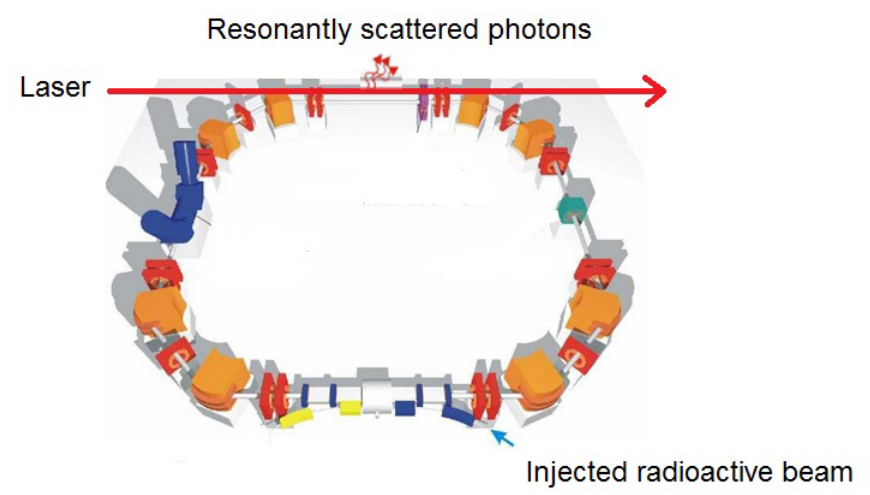

Fig. 4. Schematic showing how the laser beam interacts with the recirculating beam in the TSR.

\section{TSR layout and project planning}

A possible site for the building to house the TSR is on the west side of the ISOLDE experimental hall on the CERN site. This is illustrated in figure 5 which is an update of that given in [1]. The main disadvantage of this solution is the difference in levels of the HIE-ISOLDE beam-line and the TSR, in order to accommodate a service tunnel that runs parallel to the ISOLDE hall. This would require a tilted beam-line from the HIE-ISOLDE hall for the injection into the TSR. The figure also shows the possible positions of the external spectrometer that uses beam extracted from the TSR.

CERN's Research Board approved the TSR project in May 2012. The detailed study for costing the construction of the building, injector line, replacement power supplies, vacuum systems, beam diagnostics and other items necessary for full integration with CERN systems was completed in September 2013, but did not include the cost of the extraction line to the external spectrometer. At the time of writing, the required budget for the TSR project has not been included in CERN's Medium-Term Plan, so no timescale can be set for its completion. The UK funding agency STFC has meanwhile approved funding for both in-ring and external detector arrays (including the superconducting ex-MRI magnet), and the 4-year project to build these detectors commenced in January 2015. CERN has made provision for the additional beam-line that will allow the external spectrometer to be exploited with radioactive beams direct from HIE-ISOLDE, while waiting for additional funding to realise the CERN TSR project. 


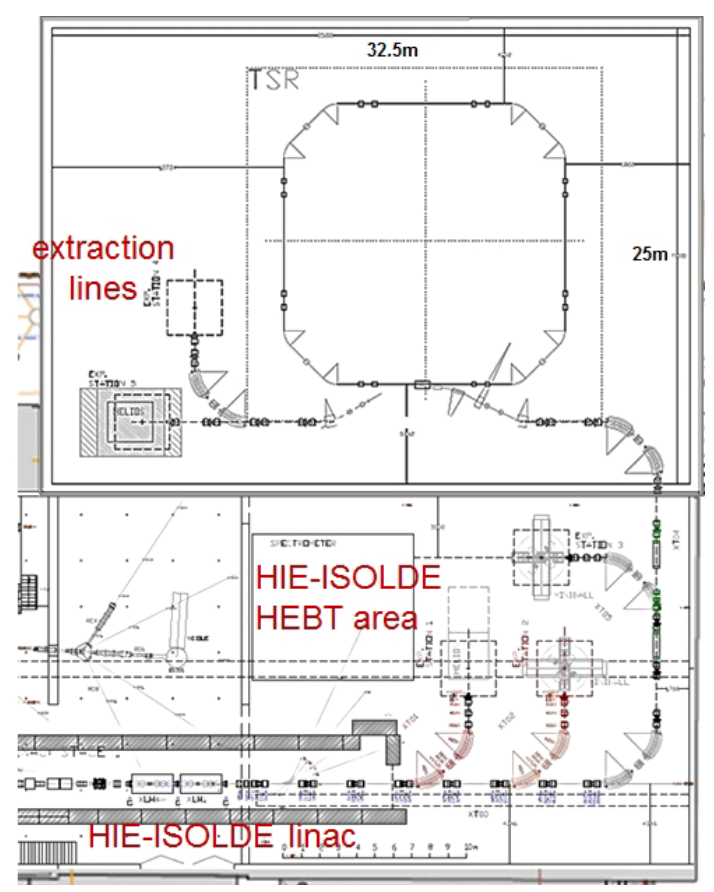

Fig. 5. Scheme of the proposed layout of the TSR at the ISOLDE facility CERN. Shown in the figure are the existing linear accelerator of HIE-ISOLDE and its High Energy Beam Transport area, as well as the possible external beam configurations for the TSR.

This work has been supported by the UK Science and Technology Facilities Council, the Max-Planck-Society, and by FWO-Vlaanderen Belgium, grants G.0706.08 "Big Science".

\section{REFERENCES}

[1] M. Grieser et al., EPJ Special Topics 207, 1 (2012).

[2] B. Mei et al., Phys. Rev. C 92, 035803 (2015).

[3] A.H. Wuosmaa et al., Nucl. Instrum. Methods A 580, 1290 (2007).

[4] J.C. Lighthall et al., Nucl. Instrum. Methods A 622, 97 (2010).

[5] B.P. Kay et al., Phys. Lett. B 658, 216 (2008).

[6] B.P. Kay et al., Phys. Rev. C 84, 024325 (2011).

[7] K.L. Jones et al., Nature 465, 454 (2010).

[8] L.P. Gaffney et al., Nature 497, 199 (2013).

[9] T.F. Thorsteinsen, K. Nybø, G. Løvhøiden, Phys. Scr. 42, 141 (1990).

[10] K. Riisager et al., Phys. Lett. B 732, 305 (2014).

[11] K. Blaum, J. Dilling, W. Nörtershäuser, Phys. Scr. T152, 014017 (2013). 